\title{
Is my event log complete? - A probabilistic approach to process mining
}

Citation for published version (APA):

Hee, van, K. M., Liu, Z., \& Sidorova, N. (2011). Is my event log complete? - A probabilistic approach to process mining. In Proceedings of the 5th IEEE International Conference on Research Challenges in Information Sciences (RCIS 2011, Gosier, Guadeloupe, May 19-21, 2011) (pp. 1-12). Institute of Electrical and Electronics Engineers. https://doi.org/10.1109/RCIS.2011.6006848

DOI:

10.1109/RCIS.2011.6006848

Document status and date:

Published: 01/01/2011

Document Version:

Publisher's PDF, also known as Version of Record (includes final page, issue and volume numbers)

Please check the document version of this publication:

- A submitted manuscript is the version of the article upon submission and before peer-review. There can be important differences between the submitted version and the official published version of record. People interested in the research are advised to contact the author for the final version of the publication, or visit the $\mathrm{DOI}$ to the publisher's website.

- The final author version and the galley proof are versions of the publication after peer review.

- The final published version features the final layout of the paper including the volume, issue and page numbers.

Link to publication

\section{General rights}

Copyright and moral rights for the publications made accessible in the public portal are retained by the authors and/or other copyright owners and it is a condition of accessing publications that users recognise and abide by the legal requirements associated with these rights.

- Users may download and print one copy of any publication from the public portal for the purpose of private study or research.

- You may not further distribute the material or use it for any profit-making activity or commercial gain

- You may freely distribute the URL identifying the publication in the public portal.

If the publication is distributed under the terms of Article 25fa of the Dutch Copyright Act, indicated by the "Taverne" license above, please follow below link for the End User Agreement:

www.tue.nl/taverne

Take down policy

If you believe that this document breaches copyright please contact us at:

openaccess@tue.nl

providing details and we will investigate your claim. 


\title{
Is My Event Log Complete? A Probabilistic Approach to Process Mining
}

\author{
Kees M. van Hee, Zheng Liu, Natalia Sidorova \\ Department of Mathematics and Computer Science \\ Eindhoven University of Technology \\ P.O. Box 513, 5600 MB Eindhoven, The Netherlands \\ \{k.m.v.hee, z.liu3, n.sidorova\}@ $\}$ tue.nl
}

\begin{abstract}
Process mining is a technique for extracting process models from event logs recorded by information systems. Process mining approaches normally rely on the assumption that the log to be mined is complete. Checking log completeness is known to be a difficult issue. Except for some trivial cases, checkable criteria for log completeness are not known. We overcome this problem by taking a probabilistic point of view. In this paper, we propose a method to compute the probability that the event log is complete. Our method provides a probabilistic lower bound for log completeness for three subclasses of Petri nets, namely, workflow nets, T-workflow nets, and S-workflow nets. Furthermore, based upon the complete log obtained by our methods, we propose two specialized mining algorithms to discover T-workflow nets and S-workflow nets, respectively. We back up our theoretical work with empirical studies that show that the probabilistic bounds computed by our method are reliable.
\end{abstract}

Keywords-process mining, workflow management, event log, Petri nets, probabilistic analysis.

\section{INTRODUCTION}

Many processes today are supported by computer systems which $\log$ their events. Originally these logs were used to trace back the events and to detect whether errors or exceptions had occurred. Today the challenge has moved to the area of deriving more sophisticated information from event logs. Process mining is a branch of data mining that is focused on the discovery of the underlying process model, i.e., the model of the process that generated the event $\log [2-8,12,13]$.

Process mining algorithms are often based on the assumption that the underlying process handles individual cases, which can be handling a claim in an insurance company, the application of a medical protocol to a patient in a hospital or the maintenance of a car in a repair shop. A log typically consists of events that can be grouped in sequences per case. For mining purposes we often assume that these cases are independent of each other, which means that the order of events per case is only determined by the characteristics of the case and not by the accidental presence of other cases. Certainly the duration of an event, waiting times and the total cycle time of a case might be influenced by the presence of other cases due to queuing phenomena, but the specific event order is not.

Given an event $\log$ and a process mining algorithm that computes a process model from this $\log$, an important question is: "Can we claim that the discovered process model is identical (actually bisimilar) to the process model that generated the events?", which requires answering the question "Is my event log complete?". This question plays an essential role in standard applications of process mining, when companies want to find out with process mining what their actual processes are, and this question becomes even more important in the domain of automated and continuous audit, when the auditing company checks that the process behaviour represented in the $\log$ complies to the rules and the auditors want to be certain that the log they have obtained contains enough information about the behaviour of the process, i.e. that their compliance check on the log implies that the process that generated the log is compliant to the rules and no uncompliant behaviour will be encountered if the organisation continues using this process.

There are some theoretical results for log completeness, i.e., $[3,10,17]$. These works are, however, based on a number of assumptions which cannot be verified in practice, for example, the assumption that every two events that may happen directly one after another according to the model implemented in the system have also occurred in the log directly one after another. One can only check this assumption if the process model is known, but this is exactly the problem of process mining, which results in the circular reasoning.

In this paper we take a probabilistic approach in order to guarantee, with some probability, that the event log is complete and the discovered process is bisimilar to the generating process. We consider each case as a random variable and each event $\log$ as a sequence of independent samples from this random variable. We first investigate the problem of log completeness in the context of the $\alpha$-algorithm [3] for process mining, which mines Petri nets, in particular workflow nets [1], as process models. We derive a probabilistic bound for log completeness for the class of structured sound workflow nets.

At the next step we try and improve the mining algorithm and come up with a more precise probabilistic bound for completeness by taking into account available information about the process structure. We consider two simple but important classes of structured sound workflow nets: S-workflows and T-workflows. While S-workflows do not support parallelism but allow modeling choices, T-workflows do the opposite: they provide support for modelling parallelism but do not 
allow for having choices in the model. S-workflows model in principle all sequential processes, which are often used for processing cases in such organisations as insurance companies, financial and governmental institutions. T-workflows are in fact standardly used as project planning models in large construction or maintenance projects in e.g. oil, building and aircraft industries.

In case we know that the process that produced the log works as an S-workflow, or a T-workflow, we can employ this information in our mining procedure. Due to the use of this information in computing the probabilistic bound, we achieve more precise estimations and we are able to claim that the log is complete with a significantly higher probability than in case we would apply the probabilistic bound we have for structured sound workflow nets to S-nets and T-nets.

The rest of this paper is organised as follows. Section II gives basic concepts from probability and set theory. Section III introduces concepts of Petri nets and process mining domains. In Section IV we introduce our probabilistic framework for process mining. In Section V we derive a probabilistic bound for log completeness for the class of structured sound workflow nets. In Section VI we define a mining algorithm and give a probabilistic bound for log completeness for Sworkflow nets. In Section VII we do it for the class of acyclic T-workflow nets. In Section VIII we empirically evaluate the quality of the probabilistic bounds for these three classes of workflow nets. Finally, Section IX discusses related works, and Section X concludes this paper.

\section{Preliminaries}

Let $S$ be a set. The powerset of $S$ is denoted by $\mathcal{P}(S)$. We use $|S|$ to denote the number of elements in $S$. The set of all natural numbers is denoted as $\mathbb{N}$. A bag (or multiset) $b$ over $S$ is a function $b: S \rightarrow \mathbb{N}$. We denote a bag by listing its elements with indications of their multiplicity witin square brackets, e.g. in a bag $\left[a, b^{2}, c\right], a$ occurs once, $b$ twice, and $c$ once. The set of all bags over $S$ is denoted by $\mathbb{B}(S)$. A set can be seen as a special kind of bag where all elements only occur once. A sequence $\sigma$ of length $l \in \mathbb{N}$ over $S$ is a function $\sigma:\{1, \ldots, l\} \rightarrow S$, which we denote as $\sigma=$ $\langle\sigma(1), \sigma(2), \ldots, \sigma(l)\rangle$. The length of a sequence is denoted by $|\sigma|$. The set of all finite sequences over $S$ is denoted as $S^{*}$.

Let $(\Omega, \mathfrak{F}, \mathbb{P})$ be a probability space, where $\Omega$ is the set of outcomes, assumed to be countable, $\mathfrak{F}=\mathbb{P}(\Omega)$, and $\mathbb{P}: \mathfrak{F} \rightarrow[0,1]$ such that $\mathbb{P}[\emptyset]=1-\mathbb{P}[\Omega]=0$, and for $F_{1}, F_{\infty}, \ldots, F_{n}$ elements of $\mathfrak{F}$ which are pairwise disjoint, $\mathbb{P}\left[\bigcup_{n=1}^{\infty} F_{n}\right]=\sum_{n=1}^{\infty} \mathbb{P}\left[F_{n}\right]$. A random variable $X$ is a function $X^{n=1}: \Omega \rightarrow R$, where $R$ is a set of values, and we define $\mathbb{P}[X \in A]=\mathbb{P}[\{\omega \in \Omega \mid X(\omega) \in A\}]$ for $A \subseteq R$. In particular $\mathbb{P}[X=x]=\mathbb{P}[\{\omega \in \Omega \mid X(\omega)=x\}]$. So we have introduced a probability measure $\pi$ on $R: \pi(x)=\mathbb{P}[X=x]$ for $x \in R$. In case $R=\mathbb{R}$, we can compute the expectation of $X$ which is $\mathbb{E}[X]=\sum_{x \in R} x \mathbb{P}[X=x]$ and the variance
$\sigma^{2}(x)=\mathbb{E}\left[X^{2}\right]-\mathbb{E}[X]^{2}$.

\section{BASIC CONCEPTS OF PETRI NETS AND PROCESS MINING}

A Petri net is a 3-tuple $N=(P, T, F)$, where $P$ and $T$ are two disjoint sets of places and transitions respectively, $F \subseteq(P \times T) \cup(T \times P)$ is a flow relation. Elements of $P \cup T$ are the nodes of $N$, elements of $F$ are the arcs. Given a node $n \in(P \cup T)$, we define its preset ${ }^{\bullet} n=\left\{n^{\prime} \mid\left(n^{\prime}, n\right) \in F\right\}$, and its postset $n^{\bullet}=\left\{n^{\prime} \mid\left(n, n^{\prime}\right) \in F\right\}$.

Markings are states of a net. A marking $m$ of a Petri net $N=(P, T, F)$ is defined as a bag over $P$. A pair $(N, m)$ is called a marked Petri net. A transition $t \in T$ is enabled in a marking $m \in \mathbb{B}(P)$ if $\bullet \leq m$. An enabled transition may fire. A transition firing results in a new marking $m^{\prime}$ where $m^{\prime}=m-\bullet t+t^{\bullet}$, denoted by $N: m \stackrel{t}{\rightarrow} m^{\prime}$. We say that $\sigma=\left\langle t_{1}, \ldots, t_{n}\right\rangle$ is a firing sequence of $(N, m)$ if $N: m \stackrel{t_{1}}{\longrightarrow}$ $m_{1} \stackrel{t_{2}}{\longrightarrow} \ldots \stackrel{t_{n}}{\longrightarrow} m_{n}$ for some markings $m_{1}, \ldots, m_{n} \in \mathbb{B}(P)$; we write then $N: m_{1} \stackrel{\sigma}{\rightarrow} m_{n}$. We write $N: m \stackrel{*}{\rightarrow} m^{\prime}$ if $N: m \stackrel{\sigma}{\rightarrow} m^{\prime}$ for some firing sequence $\sigma \in T^{*}$. The set of all reachable markings of a marked Petri net $(N, m)$ is denoted by $\mathcal{R}(N, m)=\left\{m^{\prime} \in \mathbb{B}(P) \mid N: m \stackrel{*}{\rightarrow} m^{\prime}\right\}$. The reachability graph of a Petri net is a graph representation of its possible firing sequences. It is denoted as $\mathcal{G}(N, m)=\left\{\left(m^{\prime}, t, m^{\prime \prime}\right) \mid N\right.$ : $\left.m^{\prime} \in \mathcal{R}(N, m) \wedge m^{\prime} \stackrel{t}{\rightarrow} m^{\prime \prime}\right\}$. For an elaborate introduction to Petri nets, the reader is referred to $[9,16]$.

A special class of Petri nets used for modeling workflows are workflow nets [1].

Definition 1 (Workflow net). A workflow net is a 5-tuple $N=(P, T, F, i, f)$ where $(P, T, F)$ is a Petri net, $i \in P$ is the initial place, such that ${ }^{\bullet} i=\emptyset, f \in P$ is the final place, such that $f^{\bullet}=\emptyset$, and each node $n \in P \cup T$ is on a directed path from $i$ to $f$.

The initial marking of a workflow net is [i] and the designated final marking of a workflow net is $[f]$. A firing sequence $\sigma \in T^{*}$ leading from the initial to the final marking, i.e. $N:[i] \stackrel{\sigma}{\rightarrow}[f]$, is called a trace. An important property of workflow nets is soundness [1], which means that the process always has an option to terminate:

Definition 2 (Soundness of workflow nets). A workflow net $N$ is sound if $N: m \stackrel{*}{\rightarrow}[f]$ for any marking $m \in \mathcal{R}(N,[i])$.

We further identify three special classes of workflow nets, namely, S-workflow nets, T-workflow nets, and structured workflow nets.

Definition 3 (S-workflow net). A workflow net $N$ is a $S$ workflow net if and only if $\forall t \in T,\left|t^{\bullet}\right|=\left.\right|^{\bullet} t \mid=1$.

Definition 4 (T-workflow net). A workflow net $N$ is a Tworkflow net if and only if $\forall p \in P,\left|p^{\bullet}\right|=|\bullet p| \leq 1$.

Definition 5 (Structured workflow net). A workflow net $N$ is a structured workflow net if and only if for all $(p, t) \in F$, (1) $\left|p^{\bullet}\right|>1$ implies $|\bullet t|=1$, (2) $\left.\right|^{\bullet} t \mid>1$ implies $|\bullet p|=1$. 
Note that S-workflow nets and T-workflow nets both belong to the class of structured sound workflow nets.

Workflow nets reflecting the actual behaviour of a system can be discovered from event logs, which are sets of traces, with every trace being a sequence of events for a completed process instance.

A standard technique used in process mining for finding a workflow model is the discovery of causal dependencies between the events of the system: If a task is always followed by another task it is likely that there is a causal relation between them. In order to describe these log-based ordering relations, we introduce the following notations:

Definition 6 (Log-based ordering relations). Let $L$ be an event $\log$ and $a, b$ be events.

1) $a \triangleright_{L} b$ iff $\exists \sigma \in L, i \in\{1,2, \ldots, n\}: \sigma(i)=a \wedge \sigma(i+$ $1)=b$, i.e. a directly follows $b$ in at least one trace,

2) $a \rightarrow_{L} b$ iff $a \triangleright_{L} b$ and $\neg\left(b \triangleright_{L} a\right)$, i.e. $b$ is a possible direct successor of $a$ and not another way around,

3) $a \|_{L} b$ iff $a \triangleright_{L} b$ and $b \triangleright_{L} a$, which indicates potential parallelism,

4) $a \#_{L} b$ iff $\neg\left(a \triangleright_{L} b\right)$ and $\neg\left(b \triangleright_{L} a\right)$, gives pairs of transitions that never follow each other directly, implying that direct successor relations and parallelism are not possible.

If we consider $L$ to be the set of all possible traces of the process, then we drop the subscript $L$ in Definition 6, i.e., we write $a \triangleright_{L} b$ as $a \triangleright b$. The set of all traces can contain an infinite number of traces in case of loops in the process.

The $\alpha$-algorithm (Algorithm 1) is a process mining algorithm that is able to extract a workflow model based on a complete log generated by a sound workflow net by examining the log-based ordering relations observed between tasks [3]:

Definition 7 (Complete event log). Let $N=(P, T, F, i, f)$ be a sound workflow net. Let $L$ be an event log of N. $L$ is complete if and only if (1) $\triangleright \subseteq \triangleright_{L}$, and (2) for any $t \in T$ there is a $\sigma \in L$ such that $t \in \sigma$.

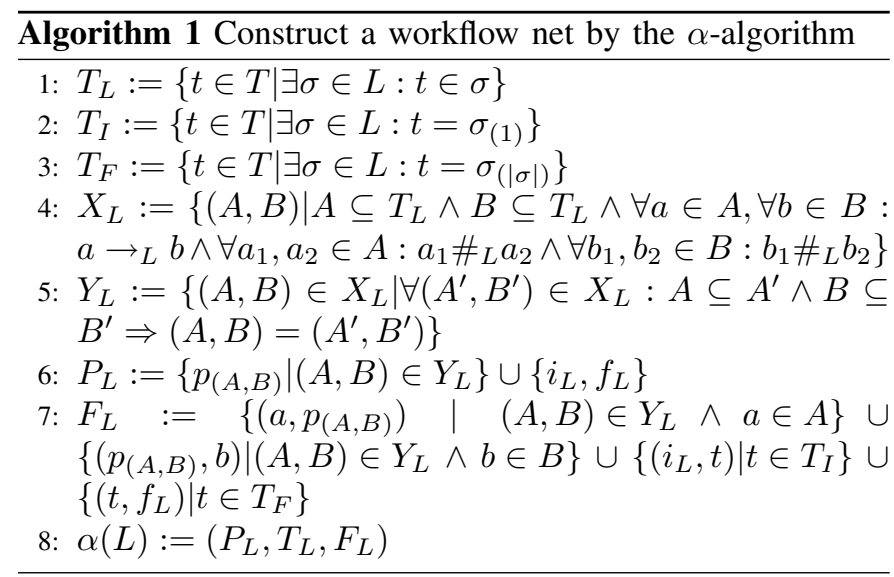

If there is a log-based ordering relation between two transitions according to the event log, then there has to be a place connecting these two transitions.
Theorem 1 (Log-based ordering relations imply connecting places [3]). Let $N=(P, T, F, i, f)$ be a sound workflow net and $L$ be a complete log. For all $a, b \in T, a \rightarrow_{L} b$ implies $a^{\bullet} \cap \bullet b \neq \emptyset$.

The following theorem shows that the $\alpha$-algorithm is able to rediscover sound structured workflow nets from complete event logs:

Theorem 2 (Rediscovering ability [3]). Let $N=$ $(P, T, F, i, j)$ be a sound structured workflow net and $L$ be a complete workflow $\log$ of $N$. If for all $a, b \in T a^{\bullet} \cap \bullet b=\emptyset$ or $b^{\bullet} \cap \bullet a=\emptyset$, then $\alpha(L)=N$ modulo renaming of places.

\section{PROBABILISTIC FRAMEWORK}

In order to handle the choice construction in Petri nets we make a realistic assumption that choices can be described by a probabilistic mechanism. In order to define a probability space, we extend the Petri net framework with a weight function $w: T \rightarrow[0,1]$ that allows to compute the probability of a transition to fire, given a marking. For a reachable marking $m$ enabling transitions $t_{1}, \ldots, t_{n}$, the probability of transition $t_{k}$ to fire $q\left(m, t_{k}\right)$ equals $w\left(t_{k}\right) /\left(\sum_{i=1}^{n} w\left(t_{i}\right)\right)$, i.e. the probability is proportional to the weight of the transition. Applying this formula to a firing sequence $\sigma=\left\langle t_{1}, \ldots, t_{n}\right\rangle$ with $m_{0} \stackrel{t_{1}}{\longrightarrow}$ $m_{1} \stackrel{t_{2}}{\longrightarrow} \ldots \stackrel{t_{n}}{\longrightarrow} m_{n}$, we derive $q(\sigma)=\prod_{i=1}^{n} q\left(m_{i-1}, t_{i}\right)$.

Next we define a probability space $(\Omega, \mathfrak{F}, \mathbb{P})$, where $\Omega=$ $\left\{\sigma \in T^{*} \mid \exists m_{1}: m_{0} \stackrel{\sigma}{\rightarrow} m_{1}\right\}$ and $m_{0}$ is the initial marking. On $\mathfrak{F}=\mathbb{P}(\Omega)$ we define the probability by $\mathbb{P}[\{\sigma\}]=q(\sigma)$. Note that each trace corresponds to a firing sequence $\sigma$ exactly. On $\Omega$ we define random variables. One of such variables is $Y: \Omega \rightarrow R$, where $R$ is the set of all $|T| \times|T|$ matrices with entries in $\{0,1\}$. For $a, b \in T$ and $1 \leq k \leq|\sigma|$,

$$
Y(\sigma)(a, b)= \begin{cases}1 & \text { if } \exists k: \sigma(k)=a \wedge \sigma(k+1)=b, \\ 0 & \text { otherwise. }\end{cases}
$$

We usually drop $\sigma$ in $Y(\sigma)$ if it causes no confusion. Thus we have a probability function $\pi$ with $\pi(y)=\mathbb{P}[Y=y]$, where $y$ is a $|T| \times|T|$ matrix over $\{0,1\}$. Note that we do not know $\pi$, we consider it as an unknown parameter.

In an event $\log L$ all the traces can be considered as samples from $Y$. If $|L|=n$ we denote them as $Y_{1}, \ldots, Y_{n}$. They are independent, identically distributed random variables, versions of $Y$.

We introduce the second random variable $Y^{n}$, the aggregation of $Y_{1}, \ldots, Y_{n}$.

$$
Y^{n}(a, b)=\bigoplus_{k=1}^{n} Y_{k}(a, b),
$$

where $\oplus$ is the logical $O R$ operator (i.e., $0+0=0$ and $0+1=$ $1+0=1+1=1)$. We call $Y^{n}$ the log characteristic matrix. Note that $Y^{n}(a, b)=1 \Leftrightarrow a \triangleright_{L} b$.

Finally, we introduce the third random variable $Z(\sigma)(a, b)$ that reflects the ordering of $a, b$ in a trace $\sigma$. For $a, b \in T$, 
$k \in\{1,2, \ldots,|\sigma|\}$, and $l \in\{1,2, \ldots,|\sigma|\}$.

$Z(\sigma)(a, b)= \begin{cases}1 & \text { if } \exists k, l: \sigma(k)=a \wedge \sigma(l)=b \wedge l>k+1 \\ 2 & \text { if } \exists k: \sigma(k)=a \wedge \sigma(k+1)=b \\ 3 & \text { if } \exists k, l: \sigma(k)=b \wedge \sigma(l)=a \wedge l>k+1 \\ 4 & \text { if } \exists k: \sigma(k)=b \wedge \sigma(k+1)=a\end{cases}$

If $|L|=n$, we consider $n$ independent, identically distributed random variables $Z_{1}, \ldots, Z_{n}$, versions of $Z$.

\section{PROBABILISTIC BOUND FOR A COMPLETE LOG}

In this section we consider logs of sound workflow nets, and we will determine a probabilistic lower bound for such a $\log$ to be complete. In Section III we have defined a $\log L$ to be complete if $\triangleright \subseteq \triangleright_{L}$ and all transitions (events) possible in the system occur in the log. If a workflow net is a structured workflow net, given its complete $\log$, the $\alpha$-algorithm will be able to reconstruct the generating net.

In Section IV, we introduced the characteristic matrix $Y(\sigma)$ for a trace $\sigma$, the characteristic matrix $Y^{n}$ over a $\log L$, and a probability $\pi$ over characteristic matrices of traces. Although $\pi$ is unknown, we know it belongs to the set of all possible probabilities $\Pi$ over the characteristic matrices for traces. Next we define a characteristic matrix of a workflow net, being a structural property of the workflow.

Definition 8 (Structural characteristic matrix). The matrixvalued function $f$ over $\Pi$ is defined by $\forall a, b \in T$,

$$
f(\pi)(a, b)= \begin{cases}1 & \text { if } q_{a b}=\sum_{y} \pi(y) y(a, b)>0 \\ 0 & \text { otherwise. }\end{cases}
$$

A $\log L$ is complete iff the characteristic matrix of the $\log$ (1) equals to the structural characteristic matrix, i.e. $Y^{n}=$ $f(\pi)$. Consequently, the probability for a $\log$ to be complete is

$$
\mathbb{P}_{\pi}\left[Y^{n}=f(\pi)\right]
$$

From (2) we can derive (see (21), (22), and (23) in Appendix)

$$
\mathbb{P}_{\pi}\left[Y^{n}=f(\pi)\right] \geq 1-\sum_{\left\{(a, b) \mid q_{a b}>0\right\}}\left(1-q_{a b}\right)^{n} .
$$

If we impose a confidence limit, then we let $(3) \geq 1-\alpha$. Hence, we get

$$
\sum_{\left\{(a, b) \mid q_{a b}>0\right\}}\left(1-q_{a b}\right)^{n} \leq \alpha .
$$

In general, we do not know $q_{a b}$. However, $q_{a b}$ can be estimated by the two following approaches.

In the first approach, we suppose that we know that for any $\pi \in \Pi$, the minimal value in $\left\{q_{a b} \mid q_{a b}>0\right\}$ is $\epsilon$, and the maximal number of $\left|\left\{(a, b) \mid q_{a b}>0\right\}\right|$ is $m$. Then (4) can be written as

$$
\sum_{k=1}^{m}(1-\epsilon)^{n}=m(1-\epsilon)^{n} \leq \alpha
$$

From (5), we can estimate a lower bound for the complete log size $n$ for a sound workflow net with certain confidence:

$$
n \geq \frac{\log \frac{\alpha}{m}}{\log (1-\epsilon)} .
$$

The second approach is based on a sample of $n$ traces in a $\log$. We consider two possible values of $q_{a b}$ :

1) The average of $Y_{n}(a, b)$, such that

$$
\bar{Y}_{n}(a, b)=\frac{1}{n} \sum_{k=1}^{n} Y_{k}(a, b) \text {. }
$$

Based on (4) and (7), we derive

$$
\sum_{\left\{(a, b) \mid \bar{Y}_{n}(a, b)>0\right\}}\left(1-\bar{Y}_{n}(a, b)\right)^{k} \leq \alpha .
$$

2) Because $Y_{n}(a, b)$ has a binomial distribution with $q_{a b}$ and $n$, we have a confidence interval for $q_{a b}$, such that

$$
q_{a b} \geq \bar{Y}_{n}(a, b)-Z_{\alpha} \sqrt{\frac{\bar{Y}_{n}(a, b)\left(1-\bar{Y}_{n}(a, b)\right)}{n}},
$$

where $Z_{\alpha}$ is the $\alpha$-percentile of the normal distribution. From (4) and (9) we conclude

$$
\sum_{\left\{(a, b) \mid \bar{Y}_{n}(a, b)>0\right\}}\left(1-\left(\bar{Y}_{n}(a, b)-Z_{\alpha} \sqrt{\left.\frac{\bar{Y}_{n}(a, b)\left(1-\bar{Y}_{n}(a, b)\right)}{n}\right)}\right)^{k} \leq \alpha .\right.
$$

In both (8) and (10), $k$ is the size of a complete log. It should be the smallest value satisfying (8) and (10), respectively. Thus we keep generating sample traces of the $\log$ until $k \leq n$, where $n$ is the sample size. This procedure is described in Algorithm 2. Such a procedure is in fact a stopping rule for a sequence of random variables $Y_{1}, Y_{2}, Y_{3}, \ldots$.

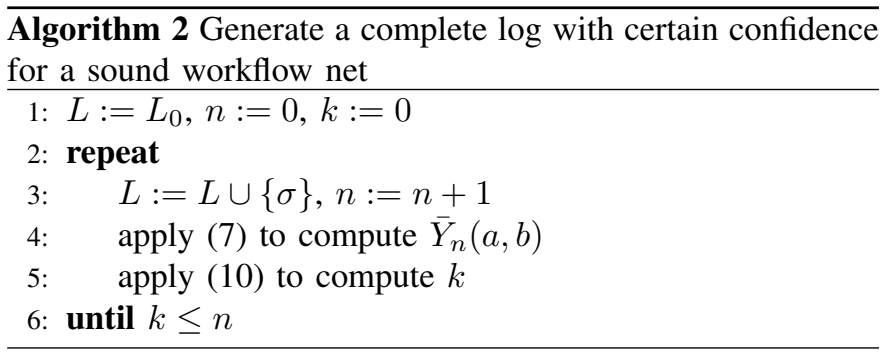

If we use (8) instead of (10), then we substitute (10) by (8) in line 5 of Algorithm 2.

We are interested in the quality of the stopping rule. We consider (8) as an estimator for (4). For a fixed log size $k$ we can compute the expectation of the estimator $\sum_{a, b}\left(1-\bar{Y}_{n}(a, b)\right)^{k}$, where the summation is over $\bar{Y}_{n}(a, b)>0$, by the following formula (see (24) in Appendix),

$$
\begin{aligned}
& \mathbb{E}_{\pi}\left[\sum_{a, b}\left(1-\delta\left(0, \bar{Y}_{n}(a, b)\right)\right)\left(1-\bar{Y}_{n}(a, b)\right)^{k}\right] \\
& =\sum_{a, b}\left(\left(1-q_{a b}\right)^{k}-\left(1-q_{a b}\right)^{n}\right) \leq \sum_{\left\{(a, b) \mid q_{a b}>0\right\}}\left(1-q_{a b}\right)^{k} .
\end{aligned}
$$

Consequently, our estimator is biased: it overestimates the confidence. However, when $n \rightarrow \infty$ the estimator is unbiased. 


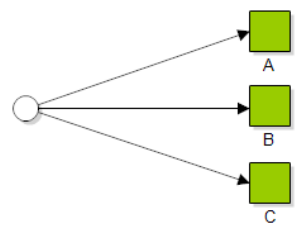

Fig. 1. A choice fragment

\section{PROBABILISTIC BOUND FOR A COMPLETE LOG OF} S-WORKFLOW NETS

In this section we consider S-workflow nets, and we determine a probabilistic lower bound for a $\log$ of an S-workflow net to be complete. As S-workflow nets are sound workflow nets (see Section III), we can use Algorithm 2 to determine the probabilistic lower bound for the size of a complete log. With a complete log, the $\alpha$-algorithm can discover the generating process. However, if we consider the structural properties of S-workflow nets, we can better estimate the probabilistic lower bounds for their complete logs.

Suppose we know the set of all transitions $T$ in an $S$ workflow net, and a lower bound $\epsilon>0$ for the probabilities of making choices in the net, i.e., $q_{t}$ for $t \in T$. For instance, in a choice fragment (Figure 1) of some S-workflow net, the probabilities to choose transitions $A, B$, and $C$ are $q_{A}, q_{B}$, and $q_{C}$, respectively, and we assume them all to be at least $\epsilon>0$. Recall that in our probabilistic framework, any $t(t \in T)$ has a weight determined by $w(t)$ (see Section IV). We assume a lower bound $l$ and an upper bound $u$ for these weights, and assume an upper bound $f$ for the fanouts $p^{\bullet}$ over $p \in P$. We derive that $q_{t} \geq \frac{l}{u f}$. Then we may choose $\epsilon=\frac{l}{u f}$.

To ensure that the log is complete, we require any transition in an S-workflow net to occur at least $k$ times in the log. Then the highest possible probability that a direct successor of a transition $t$ in the process does not occur in the $\log$ is $(1-\epsilon)^{k}$. Let $c: T \rightarrow \mathbb{N}$ be a function mapping every transitions to the number of times it occurs in the log. For a confidence limit $\alpha$ for the occurrence of an arbitrary pair of transitions in the log, we obtain: $(1-\epsilon)^{k} \leq \alpha$, Consequently, we treat a $\log$ as a complete log if all transitions occur at least $k$ times in the $\log$. This procedure is described in Algorithm 3. Once the log is complete, we can apply the $\alpha$-algorithm to reconstruct the generating net.

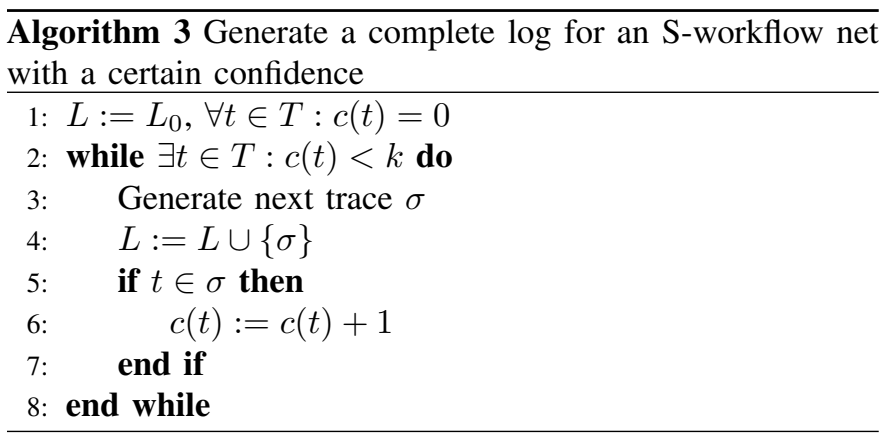

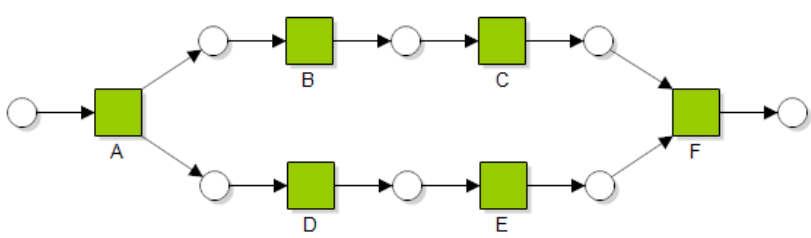

Fig. 2. An acyclic T-workflow net

\section{PRobabilistic Bound of A COMPLETE LOG FOR ACYCLIC T-WORKFLOW NETS}

In this section we propose a mining algorithm for acyclic T-workflow nets. Given an acyclic T-workflow net $N=$ $(P, T, F, i, f)$, we assume $T$ to be known. We also make an assumption on the upper bound for parallelism in $N$. Note that $|T|$ can always be taken as such a bound, but in most cases it is far too large.

As introduced in section IV, we consider a $\log L=$ $\left\langle\sigma_{1}, \sigma_{2}, \ldots \sigma_{n}\right\rangle$ to be a sample from the probability space, and $Z_{i}(a, b)=Z\left(\sigma_{i}\right)(a, b)$. We define for $a, b \in T$ predicates

1) $Q_{1}(a, b) \equiv \exists i \in\{1,2, \ldots, n\}: Z_{i}(a, b) \in\{1,2\}$,

2) $Q_{2}(a, b) \equiv \exists i \in\{1,2, \ldots, n\}: Z_{i}(a, b) \in\{3,4\}$,

3) $Q_{3}(a, b) \equiv \exists i \in\{1,2, \ldots, n\}: Z_{i}(a, b)=2$,

4) $Q_{4}(a, b) \equiv\left|\left\{i \in\{1,2, \ldots, n\} \mid Z_{i}(a, b)=2\right\}\right| \geq l$,

where $Q_{2}(a, b)=Q_{1}(b, a), Q_{3}(a, b) \Leftrightarrow a \triangleright_{L} b$, and $l$ is a parameter to be determined later in this section.

We introduce a notion of log completeness targeted at the T-workflow nets:

Definition 9 (T-complete $\log$ ). A $\log L$ of an acyclic $T$ workflow net $N$ is T-complete iff for all $a, b \in T, a \| b$ implies $Q_{1}(a, b) \wedge Q_{2}(a, b)$, and $a \rightarrow b$ implies $Q_{3}(a, b) \wedge \neg Q_{2}(a, b)$.

Note that $a \| b$ and $a \rightarrow b$ hold for all possible traces, so the subscript $L$ in Definition 6 is omitted.

Lemma 1. Completeness implies T-completeness but not vice versa.

Proof: Suppose $L$ is a complete $\log$ for an acyclic Tworkflow net $N$. By Definition 7, we have $\triangleright \subseteq \triangleright_{L}$. Since $\triangleright_{L} \subseteq$ $\triangleright$, we have $\triangleright=\triangleright_{L}$. Therefore, for all $a, b \in T$, we have $a \|$ $b \Leftrightarrow a \triangleright b, b \triangleright a \Leftrightarrow a \triangleright_{L} b$ and $b \triangleright_{L} a$ implies $Q_{1}(a, b) \wedge Q_{2}(a, b)$. Further note that $a \rightarrow b \Leftrightarrow a \triangleright b, \neg Q_{2}(a, b) \Leftrightarrow a \triangleright_{L} b$, and $\neg Q_{2}(a, b)$ implies $\Rightarrow Q_{3}(a, b) \wedge \neg Q_{2}(a, b)$. So completeness implies T-completeness.

In order to show that the converse does not hold, consider $\log L_{1}=\{\langle A, B, C, D, E, F\rangle,\langle A, D, E, B, C, F\rangle\}$ and Figure 2. For T-completeness we need $\triangleright \subseteq \triangleright_{L}$, which is not true, since $D \triangleright C$ does not hold on $L_{1}$, nor does $B \triangleright E$.

Hence, T-completeness is a weaker condition than completeness, which in fact also follows from the equivalence $a \triangleright b \equiv(a \| b) \vee(a \rightarrow b)$.

Given a T-complete $\log$ of a net $N=(P, T, F, i, f)$, we present an algorithm for mining process. The algorithm uses the fact that two tasks are connected if and only if their 
causality can be detected by inspecting the $\log$. Note that we drop the dependency on $N$ in the notation. Recall that $i$ and $j$ are the initial and final places, respectively, and we assume that $T$ is known.

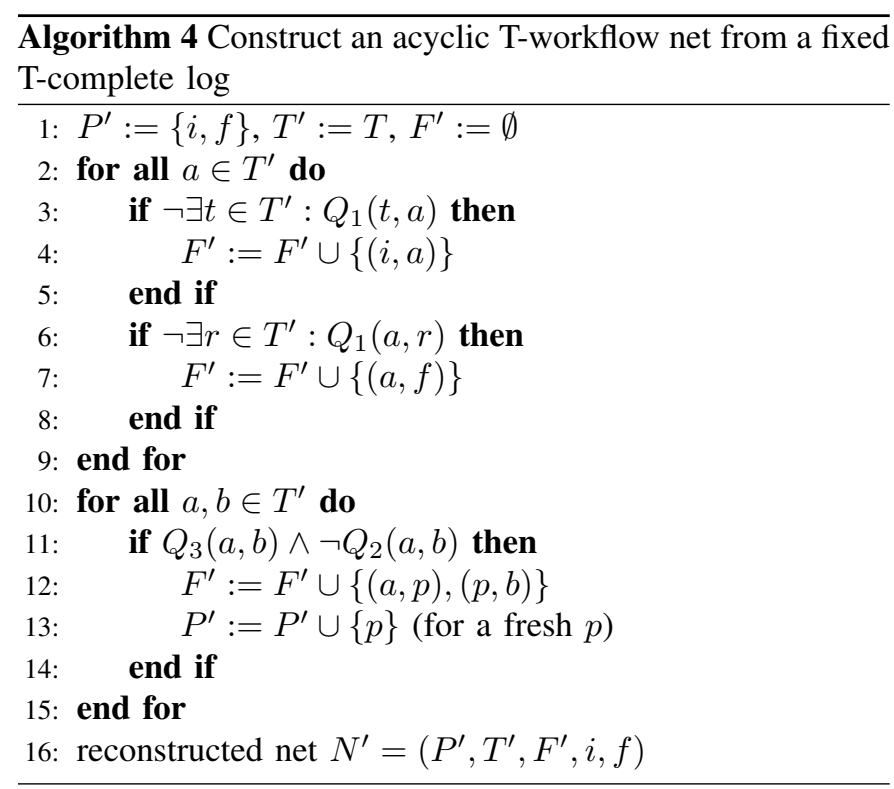

The following theorem proves that Algorithm 4 is able to reconstruct (up to bisimilarity) a generating net $N^{\prime}=$ $\left(P^{\prime}, T^{\prime}, F^{\prime}, i, f\right)$ out of a given T-complete log.

Theorem 3. Let $L$ be a T-complete log for an acyclic $T$ workflow net $N$. Then the net $N^{\prime}$ computed by Algorithm 4 is bisimilar to $N$.

Proof: Clearly, $a \triangleright_{L} b$ can only hold either in the case of $a \rightarrow b$ or in the case of $a \| b$. By Theorem $1, a \rightarrow b$ implies that there is a place between transitions $a$ and $b$. The second case is not possible due to step 4 of Algorithm 4. Therefore, $N^{\prime}$ contains a place between transitions $a$ and $b$ iff $a \rightarrow b$.

Algorithm 4 can discover the exact process model from Tcomplete logs. In most cases, however, we cannot be sure that the $\log$ is T-complete. In Algorithm 5 we propose a strategy for dealing with the logs that are possibly not T-complete: there we only add a place between two transitions $a$ and $b$ if we are certain enough that there is a causal relation between the two event, namely, $b$ never happens before $a$ in the log and $a$ happens immediately before $b$ enough times. Enough times is the parameter $l$ in $Q_{4}(a, b)$.

Algorithm 5 is able to reconstruct (up to bisimilarity) a generating net $N^{\prime}=\left(P^{\prime}, T^{\prime}, F^{\prime}, i, f\right)$ out of a given log. Our decisions in Algorithm 5, however, have a probabilistic nature and can lead to two kinds of possible errors, false positives and false negatives.

False positive is an error such that the log inspection leads to the conclusion $a \rightarrow b$, but in fact we have $a \| b$ in the underlying net. Note that if $a$ is indirectly in front of $b$ in the net (some transition $c$ lies in between), we can never conclude $a \rightarrow b$, since we will never observe $Z_{i}(a, b)=2$ because of the

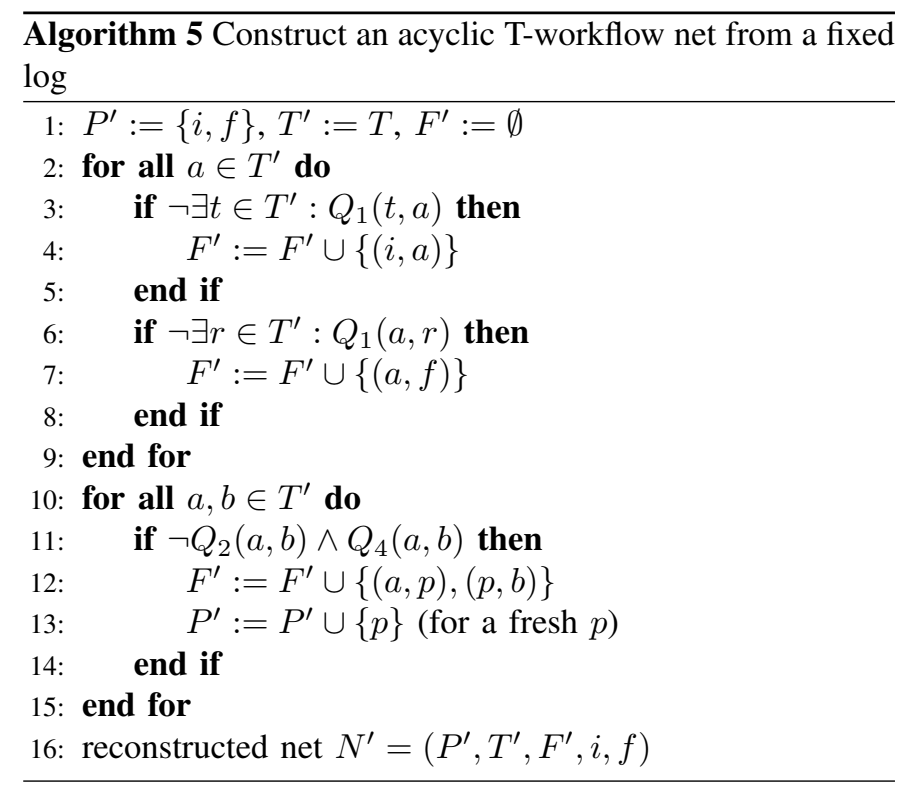

firing of other transition(s) in between. Consequently, we only have to consider the case of $a \| b$. The probability of a false positive is $\mathbb{P}_{a \| b}\left[\neg Q_{2}(a, b) \wedge Q_{4}(a, b)\right]$. An upper bound for this probability is (see (25) in Appendix for detailed derivation):

$$
\mathbb{P}_{a \| b}\left[\neg Q_{2}(a, b) \wedge Q_{4}(a, b)\right] \leq \frac{1}{2^{l}} .
$$

To ensure a confidence limit $\alpha$, we require $\frac{1}{2^{l}} \leq \alpha$. Therefore, $l$ can be computed as follows:

$$
l \geq-{ }^{2} \log \alpha .
$$

With (13), we are able to determine the minimal number $l$ of occurrences of $a \triangleright b$ in $Q_{4}(a, b)$ for Algorithm 5. For each pair $a \rightarrow b$, the probability of false positive is limited by $\alpha$.

Now, knowing a probabilistic bound for the false positive for a specific pair of transitions $(a, b)$, let us consider the set $R$ of all pairs of parallel transitions in the net, i.e. $R=\{(a, b) \in$ $T \times T \mid a \| b\}$. Note that we do not know $R$.

If the random variables $Z_{n}(a, b)$ and $Z_{n}(c, d)$, for $n=$ $1,2,3, \ldots$ and $(a, b) \neq(c, d)$, are maximally dependent, then the probability for one false positive is the same as the probability for at least one false positive for the pairs of $R$. The other extreme is that all random variables $Z_{n}(a, b)$ for $(a, b) \in R$ are independent. Then $\neg Q_{2}(a, b) \wedge Q_{4}(a, b)$ are independent events. This is the worst case as we have to investigate all the pairs. In this case, the probability of having at least one false positive can be derived using Lemma 2 (see Appendix):

$$
\begin{aligned}
& \mathbb{P}_{R}\left[\exists(a, b) \in R: \neg Q_{2}(a, b) \wedge Q_{4}(a, b)\right] \\
& =1-\left(1-\mathbb{P}_{a \| b}\left[\neg Q_{2}(a, b) \wedge Q_{4}(a, b)\right]\right)^{|R|} .
\end{aligned}
$$

Based on (12), we derive

$$
\mathbb{P}_{R}\left[\exists(a, b) \in R: \neg Q_{2}(a, b) \wedge Q_{4}(a, b)\right] \leq 1-\left(1-\frac{1}{2^{l}}\right)^{|R|} .
$$


To ensure confidence limit $\alpha$, we let $(15) \leq \alpha$. Consequently, we choose $l$ as the smallest value such that

$$
l \geq-{ }^{2} \log \left(1-(1-\alpha)^{\frac{1}{|R|}}\right) .
$$

We have to provide an estimate for the number of independent pairs in $R$. One option for such an estimate is the maximal parallelism of the process (estimated by a e.g. business analyst).

False negatives manifest themselves when the log inspection results in $Q_{1}(a, b) \wedge \neg Q_{4}(a, b)$ and we conclude that $a$ is indirectly in front of $b$ in the underlying net, while in reality we have $a \rightarrow b$ in the net. Note that given $a \rightarrow b$, we never observe $Q_{2}(a, b)$ as $b$ can never be fired before $a$. The probability for a false negative is:

$$
\mathbb{P}_{a \rightarrow b}\left[\left|\left\{i \mid Z_{i}(a, b)=2\right\}\right|<l\right]
$$

Given $a \rightarrow b$, we can only observe $Q_{1}(a, b)$ in the $\log$ (with $n$ traces), implying that either $Z_{i}(a, b)=1$ or $Z_{i}(a, b)=2$. Hence, probability (17) has a binomial distribution $B\left(n, \mathbb{P}_{a \rightarrow b}\left[Z_{i}(a, b)=2\right]\right)$. Let $p$ be $\mathbb{P}_{a \rightarrow b}\left[Z_{i}(a, b)=2\right]$, then (17) becomes:

$$
\mathbb{P}_{a \rightarrow b}\left[\left|\left\{Z_{i}(a, b)=2\right\}\right|<l\right]=\sum_{m=0}^{l-1}\left(\begin{array}{c}
n \\
m
\end{array}\right) p^{m}(1-p)^{n-m}
$$

In order to simplify the calculation in practice, we use the normal distribution for approximating (18). We obtain:

$$
\begin{aligned}
\sum_{m=0}^{l-1}\left(\begin{array}{c}
n \\
m
\end{array}\right) p^{m}(1-p)^{n-m} & =\mathbb{P}[X \leq l-1] \\
& =\mathbb{P}\left[\frac{X-n p}{\sqrt{n p(1-p)}} \leq \frac{l-1-n p}{\sqrt{n p(1-p)}}\right] \\
& \approx \mathbb{P}\left[U \leq \frac{l-1-n p}{\sqrt{n p(1-p)}}\right]
\end{aligned}
$$

Let $U$ have a standard normal distribution with zero mean and unit variance, such that $U \sim N(0,1)$. We require the probability $\mathbb{P}\left[U \leq \frac{l-1-n p}{\sqrt{n p(1-p)}}\right] \leq 0.05$. From the table of cumulative standard normal distribution [15], we get $\mathbb{P}(U \leq-1.64)=$ 0.05 . Then we have $\frac{l-1-n p}{\sqrt{n p(1-p)}}=-1.64$. Consequently, we can compute $n$ as follows.

$$
\begin{aligned}
n= & \frac{l+0.3448-1.3448 p}{p} \\
& +\frac{0.82 \sqrt{2.6896 p^{2}-(1.3792+4 l) p+4 l-1.3104}}{p}
\end{aligned}
$$

If $p$ decreases, then $n$ increases accordingly.

Note that the structural properties of the net greatly influence the size of a log that is complete with a given confidence. Here we only consider two extreme patterns:

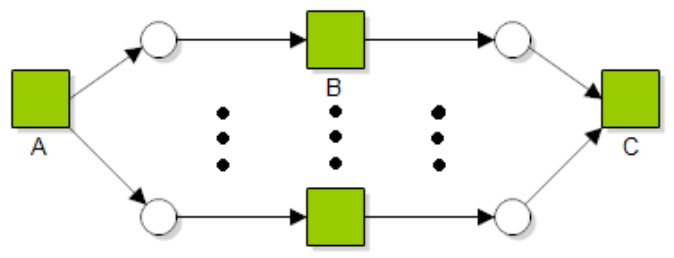

Fig. 3. Pattern 1

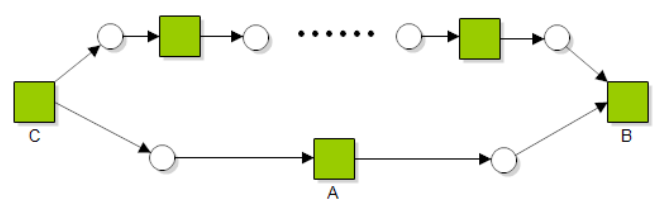

Fig. 4. Pattern 2

Pattern 1. Figure 3 shows an acyclic T-workflow net with $k$ transitions. Initial transition $A$ has $k-2$ successors $(A \rightarrow B)$. Suppose that when $A$ fires, each of the $k-2$ enabled transitions can be fired with the probability of $\frac{1}{k-2}$. Consequently, $\mathbb{P}_{A \rightarrow B}\left[Z_{i}(A, B)=2\right]=\frac{1}{k-2}$. We can estimate the $\log$ size $n$ using formula (20). For example, if $l=10$ and $k=30$, then $n \approx 430$. This implies that statistically we need just 430 traces in the log to guarantee with the $95 \%$ probability that the discovered model is the one that has generated the log.

Pattern 2. Figure 4 shows another acyclic T-workflow net with $k$ transitions. There are two parallel branches, one with $k-3$ transitions, the other one with just one transition (transition $A$ ). To discover the exact process model, we need to observe transition $A$ immediately before the final transition $B$. As transition $A$ has to be fired after all the $k-3$ transitions of the parallel branch, we derive $\mathbb{P}_{A \rightarrow B}\left[Z_{i}(A, B)=2\right]=\frac{1}{2}^{k-3}$. We use (20) to estimate $\log$ size $n$. For instance, for $l=10$ and $k=30$, then $n \approx 2.07 \times 10^{9}$. This implies that statistically we need $2.07 \times 10^{9}$ traces in a $\log$ to guarantee with the confidence of $95 \%$ that the discovered model is the one that has generated the $\log$.

We have introduced Algorithm 4 and Algorithm 5 to reconstruct an acyclic T-workflow net from a fixed $\log$ (i.e., no more new traces are added to the $\log$ ). Now we consider dynamic situations, when new traces may be added to a log until for all pairs $a, b \in T$ one of the following conditions holds: 1) $\left.\left.Q_{1}(a, b) \wedge Q_{2}(a, b), 2\right) \neg Q_{2}(a, b) \wedge \neg Q_{3}(a, b), 3\right)$ $\neg Q_{2}(a, b) \wedge Q_{4}(a, b)$. Only with this information, we can decide what kind of relation we have between transitions $a$ and $b$. We can conclude $a \|_{L} b$ (even $a \| b$ ) from condition 1 ), and $a \rightarrow{ }_{L} b$ from condition 3). Algorithm 6 generates T-complete logs for acyclic T-workflow nets in dynamic situations.

Note that in Algorithm 6, we start with a non-empty log, $\sigma$ is a new trace, and the condition in the while loop is the negation of the disjunction of the three aforementioned conditions (i.e., $\neg\left(\left(Q_{1}(a, b) \wedge Q_{2}(a, b)\right) \vee\left(\neg Q_{2}(a, b) \wedge \neg Q_{3}(a, b)\right) \vee\right.$ 


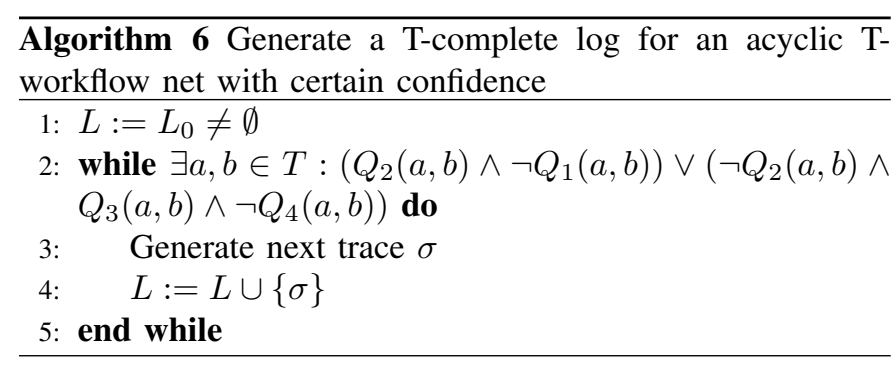

$\left.\left.\left(\neg Q_{2}(a, b) \wedge Q_{4}(a, b)\right)\right)\right)$. The loop terminates when one of the three conditions holds. Once a T-complete log has been generated, we can apply Algorithm 5 to reconstruct the generating net.

\section{EMPIRICAL RESULTS}

In order to evaluate the quality of our probabilistic bounds for log completeness empirically, we developed a software tool to generate a random benchmark set of workflow nets. Then we randomly generate a number of logs for every workflow net. Note that all traces are independent random variables and we are able to check for an arbitrary log whether this log is complete since we know the generating workflow net. Due to the limited space, we only show part of the results in this paper.

\section{A. Bounds for sound workflow nets}

First, we focus on the probabilistic bounds for log completeness obtained using Algorithm 2 both with formula (8) and with formula (10). We generate structured Jackson nets [11], which form a subset of sound workflow nets. We generated 100 structured Jackson nets, each with 30 transitions. We let $\alpha=2.5 \%$. For each of the nets we generated 100 logs. In order to compare the bounds computed using (8) and (10), each $\log$ was generated in the following way: By Algorithm 2 , we started with an empty log, and generated traces for the log. After adding a new trace, we used both (8) and (10) to check on-the-fly whether we could stop the $\log$ generation. If one formula told us to stop, then we collected the statistics of interest and continued generating traces until the second formula told us to stop as well. Then we collected the statistics again and stopped generating the log. Note that in some cases the $\log$ generation stops before we have a complete log (we do not provide $100 \%$ guarantee of log completeness), while in other cases the generation still continues when we already have a complete log, since we do not have enough confidence in the log completeness yet. In the latter case we keep the information about the minimal length of the complete log to analyze the degree of redundancy we have on average with our method.

For every generated net $N_{i}$ and every generated complete $\log l_{i j}$ (i.e., the $j^{\text {th }}$ generated complete $\log$ of $N_{i}$ ), where $i \in\{1, \ldots, k\}, j \in\{1, \ldots, m\}$, and $k=m=100$ in this experiment, we have the following test statistics:

- Number of causal pairs $\left(C_{i}\right)$ : the total number of causal pairs in net $N_{i}$,
- $\log$ size $\left(L_{i j}\right)$ : the size of the $\log l_{i j}$ generated by Algorithm 2 with either formula (8) or formula (10),

- Reliability $\left(R_{i j}\right): 1$ when $L_{i j}$ is complete, and 0 when not,

- Trace difference $\left(D_{i j}\right): D_{i j}$ is the ratio of the size of the minimal complete $\log$ forming a prefix of $l_{i j}$ to the size of $l_{i j}$.

- Missing pairs $\left(M_{i j}\right)$ : if $l_{i j}$ is not complete, then $M_{i j}$ is the ratio of the number of causal pairs which are not covered by $l_{i j}$ to the number $C_{i}$ of causal pairs.

- Log size for $80 \%$ of causal pairs $\left(E_{i j}\right)$ : the ratio of the number of traces in the minimal prefix of $l_{i j}$ which covers any $80 \%$ of all causal pairs to $L_{i j}$.

For each net $N_{i}$, we take the average $R_{i}, L_{i}, D_{i}, M_{i}, E_{i}$ over $R_{i j}, L_{i j}, D_{i j}, M_{i j}, E_{i j}$, respectively, i.e., $R_{i}=\frac{1}{m} \sum_{j=1}^{m} R_{i j}$, etc.

The empirical outcomes for (8) and (10) are listed in Table I and Table II, respectively. In both cases we used the same benchmark of workflow nets. The main conclusions we can extract are as follows.

The use of (10) instead of (8) in Algorithm 2 results in a more reliable probabilistic bound. For each net, the value of $R$ in Table $\mathrm{I}$ is never above the one in Table II. The average $R$ is increased from 0.93 (by using (8)) to 0.96 (by using (10)). Naturally, Algorithm 2 with (10) always results in a larger log than using (8).

For complete logs generated by Algorithm 2 with (8), on average all causal pairs are covered by the first $37 \%$ of all of the traces in this $\log$. The rest of the traces in the log do not contribute any more in terms of completeness. For Algorithm 2 with (10), this percentage is $31 \%$.

For incomplete logs (i.e., logs not covering all causal pairs) generated by Algorithm 2 with (8), on average, $20 \%$ of all of the causal pairs are missing in the log. For Algorithm 2 with (10), this percentage is decreased to $15 \%$.

Finally, in order to cover a majority (80\%) of all causal pairs in a net, on average we need just the first $11 \%$ of a $\log$ generated by Algorithm 2 using (8). For Algorithm 2 with (10), this percentage is $9 \%$.

\section{B. Bounds for S-workflow nets}

In the next experiment we focus on the probabilistic bounds for $\log$ completeness obtained for S-workflow nets with Algorithm 3. We generated $100 \mathrm{~S}$-workflow nets, each with 50 transitions. Note that $\mathrm{S}$-workflow nets are always sound. We take $\alpha=5 \%$, and for each of the generated nets we generate 100 logs using Algorithm 3. We use the same test statistics in Subsection VIII-A, but we naturally change the definition of Log size $\left(L_{i j}\right)$ to the size of the complete log generated by Algorithm 3. Table III presents the empirical outcomes.

The average of $R$ is 0.96 . This implies that for an $\mathrm{S}$ workflow net Algorithm results in 96 complete logs out of 100 logs, on average. In a complete log, on average the first $44 \%$ (the average of $D$ ) of all of the traces in this log cover all causal pairs, and the rest of the log does not contribute 
TABLE I

EMPIRICAL RESULTS FOR 100 STRUCTURED JACKSON NETS BY ALGORITHM 2 WITH FORMULA (8)

\begin{tabular}{|c|c|c|c|c|c|c|c|c|c|c|}
\hline & \multirow{2}{*}{$\begin{array}{c}\text { Number of } \\
\text { Causal Pairs }(C)\end{array}$} & $\begin{array}{c}\text { Reliability } \\
(R)\end{array}$ & \multicolumn{2}{|c|}{$\begin{array}{l}\text { Log Size } \\
\quad(L)\end{array}$} & \multicolumn{2}{|c|}{$\begin{array}{c}\text { Trace Difference } \\
(D)\end{array}$} & \multicolumn{2}{|c|}{$\begin{array}{c}\text { Missing Pairs } \\
(M)\end{array}$} & \multicolumn{2}{|c|}{$\begin{array}{c}\text { Log Size for } \\
80 \% \text { Causal Pairs }(E)\end{array}$} \\
\hline & & Mean & Mean & Std.Dev. & Mean & Std.Dev. & Mean & Std.Dev. & Mean & Std.Dev. \\
\hline 1 & 49 & 0.97 & 152 & 50 & 0.40 & 0.15 & 0.3265 & 0.4329 & 0.10 & 0.08 \\
\hline 2 & 382 & 1 & 1583 & 291 & 0.44 & 0.12 & 0 & 0 & 0.06 & 0.01 \\
\hline 3 & 44 & 1 & 38 & 13 & 0.40 & 0.18 & 0 & 0 & 0.13 & 0.08 \\
\hline 4 & 66 & 0.69 & 127 & 70 & 0.27 & 0.14 & 0.0161 & 0.0054 & 0.07 & 0.04 \\
\hline 5 & 58 & 0.73 & 190 & 110 & 0.28 & 0.14 & 0.0217 & 0.0111 & 0.05 & 0.03 \\
\hline 6 & 103 & 0.92 & 2246 & 834 & 0.41 & 0.14 & 0.9903 & 0 & 0.08 & 0.01 \\
\hline 7 & 41 & 0.91 & 176 & 71 & 0.38 & 0.15 & 0.9756 & 0 & 0.22 & 0.07 \\
\hline 8 & 54 & 0.95 & 233 & 60 & 0.40 & 0.13 & 0.7333 & 0.3575 & 0.23 & 0.08 \\
\hline 9 & 94 & 0.91 & 865 & 301 & 0.35 & 0.16 & 0.0130 & 0.0044 & 0.03 & 0.01 \\
\hline 10 & 89 & 0.98 & 879 & 168 & 0.42 & 0.16 & 0.0112 & 0 & 0.06 & 0.02 \\
\hline : & : & : & . & . & . & : & . & : & . & : \\
\hline 100 & 148 & 0.99 & 3047 & 621 & 0.42 & 0.14 & 0.0068 & 0 & 0.07 & 0.02 \\
\hline Avg. & 84 & 0.93 & 920 & 321 & 0.37 & 0.15 & 0.2042 & 0.0686 & 0.11 & 0.04 \\
\hline
\end{tabular}

TABLE II

EMPIRICAL RESULTS FOR 100 STRUCTURED JACKSON NETS BY ALGORITHM 2 WITH FORMULA (10)

\begin{tabular}{|c|c|c|c|c|c|c|c|c|c|c|}
\hline & \multirow{2}{*}{$\begin{array}{c}\text { Number of } \\
\text { Causal Pairs }(C)\end{array}$} & \multirow{2}{*}{$\begin{array}{c}\text { Reliability } \\
(R)\end{array}$} & \multicolumn{2}{|c|}{$\begin{array}{l}\text { Log Size } \\
(L)\end{array}$} & \multicolumn{2}{|c|}{$\begin{array}{c}\text { Trace Difference } \\
(D)\end{array}$} & \multicolumn{2}{|c|}{$\begin{array}{c}\text { Missing Pairs } \\
(M)\end{array}$} & \multicolumn{2}{|c|}{$\begin{array}{cl} & \text { Log Size for } \\
\mathbf{8 0 \%} \text { Causal Pairs }(E)\end{array}$} \\
\hline & & & Mean & Std.Dev. & Mean & Std.Dev. & Mean & Std.Dev. & Mean & Std.Dev. \\
\hline 1 & 49 & 0.99 & 184 & 50 & 0.33 & 0.14 & 0.0204 & 0 & 0.08 & 0.05 \\
\hline 2 & 382 & 1 & 1828 & 246 & 0.38 & 0.10 & 0 & 0 & 0.05 & 0.01 \\
\hline 3 & 44 & 1 & 47 & 13 & 0.33 & 0.16 & 0 & 0 & 0.11 & 0.06 \\
\hline 4 & 66 & 0.77 & 178 & 91 & 0.22 & 0.12 & 0.0152 & 0 & 0.05 & 0.03 \\
\hline 5 & 58 & 0.77 & 261 & 140 & 0.22 & 0.12 & 0.0202 & 0.0097 & 0.03 & 0.02 \\
\hline 6 & 103 & 0.97 & 2839 & 723 & 0.34 & 0.12 & 0.9903 & 0 & 0.07 & 0.03 \\
\hline 7 & 41 & 0.97 & 226 & 62 & 0.31 & 0.13 & 0.9756 & 0 & 0.17 & 0.05 \\
\hline 8 & 54 & 0.97 & 282 & 64 & 0.34 & 0.12 & 0.9136 & 0.0087 & 0.19 & 0.06 \\
\hline 9 & 94 & 0.98 & 1090 & 311 & 0.31 & 0.16 & 0.0106 & 0 & 0.02 & 0.01 \\
\hline 10 & 89 & 0.98 & 1065 & 194 & 0.35 & 0.14 & 0.0112 & 0 & 0.05 & 0.02 \\
\hline : & : & : & ${ }^{\circ}$ & ${ }^{\circ}$ & : & : & & : & : & : \\
\hline 100 & 148 & 0.99 & 3625 & 593 & 0.35 & 0.12 & 0.0068 & 0 & 0.06 & 0.02 \\
\hline Avg. & 84 & 0.96 & 1148 & 336 & 0.31 & 0.13 & 0.1519 & 0.0290 & 0.09 & 0.03 \\
\hline
\end{tabular}

TABLE III

EMPIRICAL RESULTS FOR 100 S-WORKFLOW NETS BY ALGORITHM 3

\begin{tabular}{|c|c|c|c|c|c|c|c|c|c|c|}
\hline & \multirow{2}{*}{$\begin{array}{c}\text { Number of } \\
\text { Causal Pairs }(C)\end{array}$} & \multirow{2}{*}{$\begin{array}{c}\text { Reliability } \\
(R)\end{array}$} & \multicolumn{2}{|c|}{$\begin{array}{l}\text { Log Size } \\
(L)\end{array}$} & \multicolumn{2}{|c|}{$\begin{array}{c}\text { Trace Difference } \\
(D)\end{array}$} & \multicolumn{2}{|c|}{$\begin{array}{c}\text { Missing Pairs } \\
(M)\end{array}$} & \multicolumn{2}{|c|}{$\begin{array}{l}\text { Log Size for } \\
80 \% \text { Causal Pairs }(E)\end{array}$} \\
\hline & & & Mean & Std.Dev. & Mean & Std.Dev. & Mean & Std.Dev. & Mean & Std.Dev. \\
\hline 1 & 81 & 1 & 1022 & 258 & 0.33 & 0.16 & 0 & 0 & 0.05 & 0.01 \\
\hline 2 & 137 & 0.92 & 417 & 107 & 0.62 & 0.19 & 0.0082 & 0.0024 & 0.09 & 0.02 \\
\hline 3 & 128 & 0.86 & 179 & 25 & 0.66 & 0.15 & 0.0078 & 0 & 0.14 & 0.04 \\
\hline 4 & 135 & 0.97 & 380 & 61 & 0.43 & 0.16 & 0.0074 & 0 & 0.08 & 0.02 \\
\hline 5 & 86 & 0.86 & 263 & 56 & 0.55 & 0.22 & 0.0116 & 0 & 0.07 & 0.02 \\
\hline 6 & 84 & 1 & 671 & 126 & 0.25 & 0.1 & 0 & 0 & 0.04 & 0.01 \\
\hline 7 & 95 & 1 & 1833 & 383 & 0.26 & 0.12 & 0 & 0 & 0.04 & 0.01 \\
\hline 8 & 91 & 0.98 & 1163 & 214 & 0.49 & 0.19 & 0.011 & 0 & 0.06 & 0.02 \\
\hline 9 & 88 & 1 & 276 & 54 & 0.44 & 0.15 & 0 & 0 & 0.1 & 0.03 \\
\hline 10 & 136 & 0.82 & 577 & 71 & 0.72 & 0.13 & 0.0086 & 0.0027 & 0.17 & 0.03 \\
\hline : & : & : & : & : & : & : & . & : & : & . \\
\hline 100 & 106 & 1 & 853 & 156 & 0.30 & 0.14 & 0 & 0 & 0.04 & 0.01 \\
\hline Avg. & 95 & 0.96 & 847 & 153 & 0.44 & 0.16 & 0.0098 & 0.0004 & 0.07 & 0.02 \\
\hline
\end{tabular}


anymore in terms of log completeness. For an incomplete log, on average just 1 causal pair (the average of $M$ ) is missing in the log. On average, the first $7 \%$ of all of the traces in a log cover a majority $(80 \%)$ of all causal pairs in a net. Therefore, we conclude that the probabilistic bound for log completeness given by Algorithm 3 has a good quality.

We compared the results obtained for the S-nets using Algorithm 2 and using Algorithm 3. Algorithm 2 required the construction of logs that were on average $20 \%$ longer than Algorithm 3. Note that with algorithm 3 we achieve the targeted reliability of 0.95 -it is 0.96 on average on our benchmark. Thus we succeeded in improving the probabilistic bound for the log size, taking into account the net structure indeed.

\section{Bounds for acyclic T-workflow nets}

In the last experiment, we concentrate on the probabilistic bound obtained with Algorithm 6 for acyclic T-workflow nets. We generated 100 acyclic T-workflow nets, each with 30 transitions. We take $l=10$. We generate $100 \operatorname{logs}$ by Algorithm 6 for each of the generated nets. For every generated net $N_{i}$ and every generated T-complete $\log l_{i j}$, where $i \in\{1, \ldots, k\}, j \in\{1, \ldots, m\}$, and $k=m=100$ in this experiment, we have the following test statistics:

- Number of direct successor pairs $\left(S_{i}\right)$ : the total number of pairs of transitions that have the direct successor relation (Definition 6) in $N_{i}$,

- $\log$ size $\left(L_{i j}\right)$ : the $\log$ size for $l_{i j}$ computed with Algorithm 6,

- False positive $\left(A_{i j}\right)$ : the number of pairs of transitions that do not have direct successor relation in reality the net but considered to have direct successor relation in $l_{i j}$ by Algorithm 6,

- False negative $\left(B_{i j}\right)$ : the number of pairs of transitions that do have direct successor relation in the net but considered not to have it in $l_{i j}$ by Algorithm 6,

- Reliability $\left(R_{i j}\right)$ : whether or not $l_{i j}$ covers exactly the direct successor pairs of $N_{i}$, i.e. it is both false positive free and false negative free.

For each net $N_{i}$, we compute the averages $R_{i}, L_{i}, A_{i}, B_{i}$ of $R_{i j}, L_{i j}, A_{i j}, B_{i j}$ over $j$, respectively, i.e., $R_{i}=\frac{1}{m} \sum_{j=1}^{m} R_{i j}$, etc.

The empirical outcomes are shown in Table IV. The average result of $R$ is 0.93 , which is above 0.90 we aimed at in this series of experiments. The average result of $A(0.016)$ is much smaller than the average result of $B$ (1.166). This implies that if a log generated by Algorithm 6 is not complete, then it is much more likely that the log misses some direct successor pairs (a false negative) than that that we erroneously diagnose pairs as being in the direct successor relation (a false positive).

We compared the results obtained for the T-nets using Algorithm 2 and by Algorithm 5. Algorithm 2 required the construction of logs that were on average 6 times longer than Algorithm 5. Note that with algorithm 5 we achieved the targeted reliability (and even overperformed it) on average. Thus taking into account the information about the structure of T-workflow nets significantly improves the probabilistic bound for the $\log$ size.

We also observe a number of outliers with respect to the Reliability $(R)$. Consider the $9^{\text {th }}$ net in Table IV as such an example. For this net only 52 of the 100 generated logs are complete, and in every of the 48 incomplete logs there is exactly one direct successor pair missing. By inspecting the net, we found a variant of Pattern 2 for false negative (see Section VII). Figure 5 shows a part of net 9 and highlights this pattern in a black rectangle. As $l$ is 10 and there are 13 transitions in this highlighted subset, from (20) we get $n \approx 15812$. This implies that in theory we need a log with a minimal size of 15812 to determine $t_{29} \rightarrow t_{23}$. On the other hand, our empirical results show that on average the log size for net 9 is 7962 (standard deviation is 7707). Our assumptions for this net are thus overoptimistic. Consequently, $t_{29} \rightarrow t_{23}$ cannot be determined from many logs, resulting in incomplete logs. The same pattern was observed in all other outliers.

Interestingly, this pattern reflects the situation that can also be observed in real life. When one of the parallel branches is shorter then the other(s) (possibly not in the terms of the number of transitions but in the sense of execution time), the probability to observe the pair of type $(A, B)$ (see Figure 4$)$ is extremely low, or even 0 (when a timed process is considered).

\section{RELATED WORKS}

In [5], a finite state machine method, a neural network method, and a Markov approach were proposed for process discovery in case of software engineering processes. These methods concentrated on sequential processes. The methods were extended in [6] to detect concurrent processes by a number of specific metrics, i.e., event type counts, periodicity and causality. In [13] and [12], a hidden Markov model was employed in the context of workflow management, in the case of sequential processes and concurrent processes, respectively. In the works aforementioned, the focus was on identifying the dependency relations between events.

It is obvious that the mining algorithms proposed in this paper are adopted from control-flow mining algorithms, i.e., Alpha algorithm. In [14] a method for genetic process mining was proposed. Such a process mining algorithm is capable of discovering all common control-flow structures (i.e. sequences, choices, parallelism, loops, non-free-choices, invisible tasks, and duplicate tasks as well) while being robust to noisy logs. This method, however, has more difficulties to mine models with constructs that allow for many interleaving situations. These two algorithms both assume the event log is complete.

In [17] and [10], a heuristic mining approach and a fuzzy mining approach were proposed, respectively. They both employs heuristics to limit the set of precedence relations included in a model. The heuristic miner is a practical applicable mining algorithm that can handle noise. It includes three steps: construction of dependency graph, construction of input and output expressions for each activity, and search for long 
TABLE IV

EMPIRICAL RESULTS FOR 100 ACYCLIC T-WORKFLOW NETS BY ALGORITHM 6

\begin{tabular}{|c|c|c|c|c|c|c|c|c|}
\hline & \multirow{2}{*}{$\begin{array}{c}\text { Number of } \\
\text { Successor Pairs }(S)\end{array}$} & $\begin{array}{c}\text { Reliability } \\
(R)\end{array}$ & \multicolumn{2}{c|}{$\begin{array}{c}\text { Log Size } \\
(\end{array}$} & Mean & Mean & Std.Dev. & \multicolumn{2}{c|}{ Mean Positive } & \multicolumn{2}{c|}{$\begin{array}{c}\text { False Negative } \\
(B)\end{array}$} \\
\cline { 3 - 8 } & Direct Succ.Dev. & Mean & Std.Dev. \\
\hline 1 & 35 & 0.98 & 1803 & 684 & 0 & 0 & 1.5 & 0.5 \\
2 & 36 & 0.99 & 2201 & 645 & 0 & 0 & 2 & 0 \\
3 & 36 & 0.94 & 4878 & 2544 & 0 & 0 & 1 & 0 \\
4 & 37 & 1 & 9589 & 4243 & 0 & 0 & 0 & 0 \\
5 & 38 & 1 & 2514 & 2360 & 0 & 0 & 0 & 0 \\
6 & 37 & 1 & 3067 & 1004 & 0 & 0 & 0 & 0 \\
7 & 37 & 0.99 & 1862 & 1280 & 0 & 0 & 1 & 0 \\
8 & 34 & 0.99 & 845 & 403 & 0 & 0 & 1 & 0 \\
9 & 32 & 0.52 & 7962 & 7707 & 0 & 0 & 1 & 0 \\
10 & 37 & 0.97 & 14925 & 5160 & 0.33 & 0.47 & 1.33 & 0.47 \\
$\vdots$ & $\vdots$ & $\vdots$ & $\vdots$ & $\vdots$ & $\vdots$ & $\vdots$ & $\vdots$ \\
100 & 37 & 0.86 & 4333 & 2085 & 0 & 0 & 1 & 0 \\
\hline Avg. & $\mathbf{3 5}$ & $\mathbf{0 . 9 3}$ & $\mathbf{1 1 6 4 8}$ & $\mathbf{6 5 2 1}$ & $\mathbf{0 . 0 1 6}$ & $\mathbf{0 . 0 1 8 5}$ & $\mathbf{1 . 1 6 6}$ & $\mathbf{0 . 0 7 8 8}$ \\
\hline
\end{tabular}

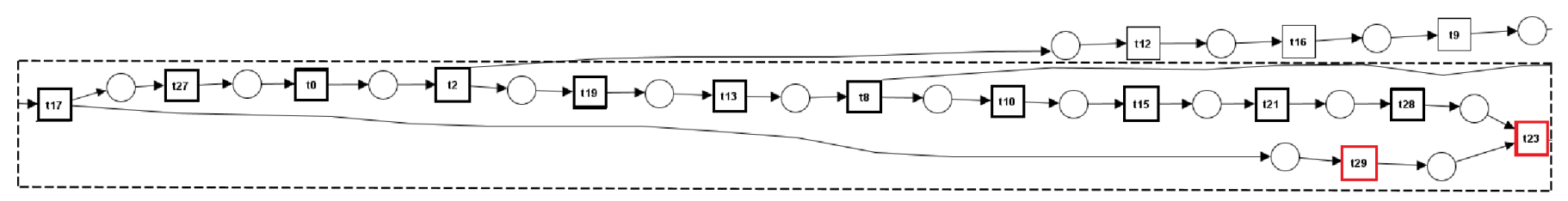

Fig. 5. Part of net 9 resulting in low reliability

distance dependency relations. The fuzzy miner assumes that problems in mining large scale processes are caused by mismatch between fundamental assumptions of traditional process mining and the characteristics of real-life processes. In this approach, the authors developed an adaptive simplification and visualization technique for process models, which is based on two metrics, significance and correlation. The two metrics are similar to the concept of data clustering domain where a binary distance metric is inferred to find related subsets of attributes. Significance can be determined both for tasks and precedence relations over them. It measures the relative importance of behaviour. As such, it specifies the level of interest in tasks and their control dependency. Correlation is only relevant for precedence relations over tasks, which measures how closely related two events following one another. The heuristic miner and the fuzzy miner only express the main behaviours registered in an event log. They lose absolute precision and cannot describe the complete behaviours.

\section{CONCLUSION}

In this paper, we address the problem of log completeness in the context of process mining. Our method give a probabilistic bound of log completeness, also implying the same probabilistic bound that the model discovered on the process $\log$ is an exact representation of the process that has generated this $\log$. We considered three classes of nets: sound structured workflow nets, S-workflow nets, and T-workflow nets. While for the first two classes we use the $\alpha$-algorithm for model discovery, we propose a new algorithm for the discovery of
T-workflow nets. In our empirical studies, we showed that our method provides the promised quality of model discovery.

In the future we plan to find patterns of "dangerous" net structures (like our Pattern 2) that would allow us to improve the probabilistic bounds for the subclasses that currently manifest themselves as outliers. We are also interested in "positive" patterns, which would allow us to lower the probabilistic bounds for nets matching these patterns, since our probabilistic bound can clearly be further lowered for some classes of nets. We also want to investigate the applicability of Bayesian methods for the $\log$ completeness problem

\section{REFERENCES}

[1] W.M.P. van der Aalst. The Application of Petri Nets to Workflow Management. The Journal of Circuits, Systems and Computers, 8(1):21-66, 2001.

[2] W.M.P. van der Aalst, B.F. van Dongen, J. Herbst, L. Maruster, G. Schimm, and A.J.M.M. Weijters. Workflow Mining: A Survey of Issues and Approaches. Data and Knowledge Engineering, 47(2):237-267, 2003.

[3] W.M.P. van der Aalst, A.J.M.M. Weijters, and L. Maruster. Workflow Mining: Discovering Process Models from Event Logs. IEEE Transactions on Knowledge and Data Engineering, 16(9):1128-1142, 2004.

[4] R. Agrawal, D. Gunopulos, and F. Leymann. Mining Process Models from Workflow Logs. In Proceedings of 6th International Conference on Extending Database Technology, pages 469-483, 1998

[5] J.E. Cook and A.L. Wolf. Discovering Models of Software Processes from Event-Based Data. ACM Transactions on Software Engineering and Methodology, 7(3):215-249, 1998.

[6] J.E. Cook and A.L. Wolf. Event-Based Detection of Concurrency. In Proceedings of 6th International Symposium on the 
Foundations of Software Engineering (FSE-6), pages 35-45, Orlando, FL, November 1998.

[7] J.E. Cook and A.L. Wolf. Software Process Validation: Quantitatively Measuring the Correspondence of a Process to a Model. ACM Transactions on Software Engineering and Methodology, 8(2):147-176, 1999.

[8] A. Datta. Automating the Discovery of As-Is Business Process Models: Probabilistic and Algorithmic Approaches. Information Systems Research, 9(3):275-301, 1998.

[9] C. Girault and R. Valk, editors. Petri Nets for System Engineering: A Guide to Modelling, Verification, and Applications. Springer-Verlag, Berlin, 2003.

[10] C.W. Gunther and W.M.P. van der Aalst. Fuzzy mining: adaptive process simplification based on multi-perspective metrics. In Proceedings of 5th International Conference on Business Process Management, pages 328-343, Brisbane, Australia, 2007.

[11] K.M. van Hee, J. Hidders, G. Houben, J. Paredaens, and P. Thiran. On the Relationship between Workflow Models and Document Types. Information Systems, 34(1):178-208, 2008.

[12] J. Herbst. Dealing with Concurrency in Workflow Induction. In Proceedings of European Concurrent Engineering Conference. SCS Europe, Gent, Belgium, 2000.

[13] J. Herbst. A Machine Learning Approach to Workflow Management. In Proceedings of 11th European Conference on Machine Learning, volume 1810 of Lecture Notes in Computer Science, pages 183-194, Berlin, 2000. Spinger-Verlag.

[14] A.K. Alves de Medeiros, A.J.M.M. Weijters, and W.M.P. van der Aalst. Genetic process mining: A basic approach and its challenges. In BPM 2005 Workshops (Workshop on Business Process Intelligence, volume 3812 of Lecture Notes in Computer Science, pages 203-215, Springer-Verlag, Berlin, 2006.

[15] D.C. Montgomery and G.C. Runger. Applied Statistics and Probability for Engineers. John Wiley \& Sons, 5 edition, 2011.

[16] T. Murata. Petri Nets: Properties, Analysis and Applications. Proceedings of the IEEE, 77(4):541-580, April 1989.

[17] A.J.M.M. Weijters and A.K. Alves de Medeiros. Process mining with the HeuristicsMiner algorithm. 2006.

\section{APPENDIX}

In this final part we present a lemma and detailed derivation of (3), (11), (12) in this paper.

Lemma 2. $\mathbb{P}\left[\exists i \in\{1,2, \ldots, n\}: A_{i}\right]=1-\left(1-\mathbb{P}\left[A_{i}\right]\right)^{n}$, where $A_{i}$ are independent events.

Proof:

$$
\begin{aligned}
\mathbb{P}\left[\exists i \in\{1, \ldots, n\}: A_{i}\right] & =1-\mathbb{P}\left[\forall i \in\{1, \ldots, n\}: \neg A_{i}\right] \\
& =1-\prod_{i=1}^{n} \mathbb{P}\left[\neg A_{i}\right]=1-\left(1-\mathbb{P}\left[A_{i}\right]\right)^{n}
\end{aligned}
$$

Formulae (21), (22), and (23) derive (3).

$$
\begin{aligned}
& Y^{n}=f(\pi) \\
& \equiv \forall a, b: f(\pi)(a, b)=0 \Rightarrow Y^{n}(a, b)=0 \\
& \quad \wedge \forall a, b: Y^{n}(a, b)=0 \Rightarrow f(\pi)(a, b)=0 \\
& \equiv \forall a, b: Y^{n}(a, b)=0 \Rightarrow f(\pi)(a, b)=0 \\
& \equiv \neg\left(\neg \forall a, b: Y^{n}(a, b)=0 \Rightarrow f(\pi)(a, b)=0\right) \\
& \equiv \neg\left(\exists a, b: \neg\left(Y^{n}(a, b)=1 \vee f(\pi)(a, b)=0\right)\right) \\
& \equiv \neg\left(\exists a, b: Y^{n}(a, b)=0 \wedge f(\pi)(a, b)=1\right)
\end{aligned}
$$

$$
\begin{aligned}
& \mathbb{P}_{\pi}\left[Y^{n}=f(\pi)\right] \\
& =\mathbb{P}_{\pi}\left[\neg\left(\exists a, b: Y^{n}(a, b)=0 \wedge f(\pi)(a, b)=1\right)\right] \\
& =1-\mathbb{P}_{\pi}\left[\exists a, b: Y^{n}(a, b)=0 \wedge f(\pi)(a, b)=1\right] \\
& \geq 1-\sum_{a, b} \mathbb{P}_{\pi}\left[Y^{n}(a, b)=0 \wedge f(\pi)(a, b)=1\right] \\
& \mathbb{P}_{\pi}\left[Y^{n}(a, b)=0 \wedge f(\pi)(a, b)=1\right] \\
& = \begin{cases}\mathbb{P}_{\pi}\left[Y^{n}(a, b)=0\right] & \text { if } \pi \text { with } f(\pi)(a, b)=1 \\
0 & \text { if } \pi \text { with } f(\pi)(a, b)=0\end{cases} \\
& = \begin{cases}\prod_{k=1}^{n} \mathbb{P}_{\pi}\left[Y_{k}(a, b)=0\right] & \text { if } f(\pi)(a, b)=1 \\
0 & \text { otherwise }\end{cases} \\
& = \begin{cases}\left(1-q_{a b}\right)^{n} & \text { if } q_{a b}>0 \\
0 & \text { otherwise }\end{cases}
\end{aligned}
$$

Formula (24) derives (11).

$$
\begin{aligned}
& \mathbb{E}_{\pi}\left[\sum_{a, b}\left(1-\delta\left(0, \bar{Y}_{n}(a, b)\right)\right)\left(1-\bar{Y}_{n}(a, b)\right)^{k}\right] \\
& =\sum_{a, b}\left(\mathbb{E}_{\pi}\left[\left(1-\bar{Y}_{n}(a, b)\right)^{k}\right]-\mathbb{E}_{\pi}\left[\delta\left(0, \bar{Y}_{n}(a, b)\right)\left(1-\bar{Y}_{n}(a, b)\right)^{k}\right]\right) \\
& =\sum_{a, b}\left(\mathbb{E}_{\pi}\left[\left(1-\bar{Y}_{n}(a, b)\right)^{k}\right]-\mathbb{P}_{\pi}\left[\bar{Y}_{n}(a, b)=0\right]\right)
\end{aligned}
$$

(due to Jensen's inequality, we derive)

$$
\begin{aligned}
& \geq \sum_{a, b}\left(\left(1-\mathbb{E}_{\pi}\left[\bar{Y}_{n}(a, b)\right]\right)^{k}-\mathbb{P}_{\pi}\left[\bar{Y}_{n}(a, b)=0\right]\right) \\
& =\sum_{a, b}\left(\left(1-q_{a b}\right)^{k}-\mathbb{P}_{\pi}\left[\bigcap_{l=1}^{n} Y_{n}(a, b)=0\right]\right) \\
& =\sum_{a, b}\left(\left(1-q_{a b}\right)^{k}-\left(1-q_{a b}\right)^{n}\right) \leq \sum_{\left\{(a, b) \mid q_{a b}>0\right\}}\left(1-q_{a b}\right)^{k}
\end{aligned}
$$

Formula (25) derives (12).

$$
\begin{aligned}
& \mathbb{P}_{a \| b}\left[\neg Q_{2}(a, b) \wedge Q_{4}(a, b)\right] \\
&=\mathbb{P}_{a \| b}\left[\left|\left\{i \mid Z_{i}(a, b) \in\{3,4\}\right\}\right|=0 \wedge\left|\left\{i \mid Z_{i}(a, b)=2\right\}\right| \geq l\right] \\
& \leq \mathbb{P}_{a \| b}\left[\left|\left\{i \mid Z_{i}(a, b)=4\right\}\right|=0 \wedge\left|\left\{i \mid Z_{i}(a, b)=2\right\}\right| \geq l\right] \\
&=\sum_{m=l}^{n} \mathbb{P}_{a \| b}\left[\left|\left\{i \mid Z_{i}(a, b)=4\right\}\right|=0 \wedge\left|\left\{i \mid Z_{i}(a, b)=2\right\}\right|=m\right] \\
&=\sum_{m=l}^{n} \mathbb{P}_{a \| b}\left[\left|\left\{i \mid Z_{i}(a, b)=4\right\}\right|=0 \wedge\left|\left\{i \mid Z_{i}(a, b) \in\{2,4\}\right\}\right|=m\right] \\
&=\sum_{m=l}^{n} \mathbb{P}_{a \| b}\left[\left|\left\{i \mid Z_{i}(a, b)=4\right\}\right|=0||\left\{i \mid Z_{i}(a, b) \in\{2,4\}\right\} \mid=m\right] \\
&\left.\quad \times \mathbb{P}_{a \| b}\left[\left|\left\{i \mid Z_{i}(a, b) \in\{2,4\}\right\}\right|=m\right]\right) \\
&=\sum_{m=l}^{n} \frac{1}{2^{m}} \mathbb{P}_{a \| b}\left[\left|\left\{i \mid Z_{i}(a, b) \in\{2,4\}\right\}\right|=m\right] \\
& \leq \frac{1}{2^{l}} \sum_{m=l}^{n} \mathbb{P}_{a \| b}\left[\left|\left\{i \mid Z_{i}(a, b) \in\{2,4\}\right\}\right|=m\right]=\frac{1}{2^{l}}
\end{aligned}
$$

Note that we assume in an acyclic T-workflow net, choices of all the enabled transitions are made with equal probabilities. Therefore, $a$ and $b$ are simultaneously enabled if $Z_{i}(a, b)=\{3,4\}$, and one transition is chosen with probability $\frac{1}{2}$. 